\title{
Quantifizierung lokaler Grundwassereintritte in die Spree und deren Bedeutung für die Verockerungsproblematik in der Laustiz
}

\author{
Sven Frei ${ }^{1}$ (D) Fabian Wismeth ${ }^{2} \cdot$ Benjamin Silas Gilfedder $^{2}$ \\ Eingegangen: 31. Oktober 2019 / Überarbeitet: 6. Februar 2020 / Online publiziert: 23. Mai 2020 \\ (c) Der/die Autor(en) 2020
}

\section{Zusammenfassung}

Eine unbekannte, schwer zu bestimmende aber zentrale Komponente in der Verockerungs-Problematik der Spree ist der lokale Grundwasserzufluss. Als Teil dieser Studie wurden mithilfe des natürlichen Tracers Radon ( $\left.{ }^{222} \mathrm{Rn}\right)$ die lokalen Grundwasserzuflüsse in die Spree und Kleine Spree im Lausitzer Braunkohlerevier bestimmt. Der gesamte Grundwasserzufluss, für das $20 \mathrm{~km}$ lange Teilstück der Kleinen Spree und den $34 \mathrm{~km}$ langen Abschnitt der Spree, variierte je nach Messkampagne zwischen $\sim 3.000$ und $\sim 7.000 \mathrm{~m}^{3} \mathrm{~d}^{-1}$ (Kleine Spree) sowie $\sim 20.000$ und $\sim 38.000 \mathrm{~m}^{3} \mathrm{~d}^{-1}$ (Spree). Entlang der Spreewitzer Rinne, einem vom Tagebauabraum geprägten Aquifer, wurden Flussabschnitte mit besonders hohem, präferenziellem Grundwassereintritt identifiziert (bis zu 70\% des gesamten Zustromes). Für diese Bereiche gelangen große Mengen an gelöstem Eisen aus dem Grundwasser in die Fließgewässer. Basierend auf gemessenen lokalen Eisen- und Sulfatfrachten in beiden Fließgewässern, wurde für das Einzugsgebiet die Menge an zurückgehaltenem Eisen quantifiziert. Für das gesamte untersuchte Einzugsgebiet der Spree liegt die Menge an zurückgehaltenem Eisen durch die Eisenhydroxid-Bildung bei bis zu 120 Tonnen/Tag.

\section{Mapping and quantifying groundwater inflow to the Spree River (Lusatia) and its role in Fe precipitation and coating of the river bed}

\begin{abstract}
Local groundwater inflow is an unknown but central component for the precipitation and accumulation of iron in the Spree River, Lusatia. In this study, the natural tracer radon was used to map and quantify local groundwater inflows into the Spree and Kleine Spree rivers in the Lusatian lignite mining district. During two measurement campaigns, the total groundwater inflow for a $20 \mathrm{~km}$ long reach of the Kleine Spree and a $34 \mathrm{~km}$ long reach of the Spree ranged between $\sim 3,000$ and $\sim 7,000 \mathrm{~m}^{3} \mathrm{~d}^{-1}$ (Kleine Spree) and between $\sim 20,000$ and $\sim 38,000 \mathrm{~m}^{3} \mathrm{~d}^{-1}$ (Spree). Particularly high groundwater inflow was identified (up to $70 \%$ of total inflow) along the Spreewitzer Rinne, a local aquifer consisting of excavated mining materials. Along these river reaches, large amounts of dissolved iron are entering the rivers with inflowing groundwater.
\end{abstract}

Zusatzmaterial online Zusätzliche Informationen sind in der Online-Version dieses Artikels (https://doi.org/10.1007/ s00767-020-00452-0) enthalten. Das Zusatzmaterial enthält 1) zusätzliches Kartenmaterial, auf dem die beprobten Grundwassermessstellen entlang der Fließgewässer (Spree und Kleine Spree) verzeichnet sind, 2) eine detaillierte Beschreibung der Aufbereitung und Analyse der wasserchemischen Proben, 3) Abflussdaten für die Spree und Kleine Spree, 4) Wasserbilanzierung unter Berücksichtigung der quantifizierten Grundwasserzuflussraten, 5) Fotoaufnahmen von beiden Fließgewässern an ausgewählten Messpunkten und 6) eine detaillierte Auflistung der chemischen Messdaten.
Sven Frei

sven.frei@uni-bayreuth.de

1 Lehrstuhl für Hydrologie, Bayreuther Zentrum für Ökologie und Umweltforschung (BAYCEER), Universität Bayreuth, Bayreuth, Deutschland

2 Limnologische Station, Bayreuther Zentrum für Ökologie und Umweltforschung (BAYCEER), Universität Bayreuth, Bayreuth, Deutschland 
Using the measured iron and sulphate loadings, we calculated that up to 120 tons/day of iron (oxy)-hydroxide was retained in the combined Spree and Klein Spree catchments.

Keywords Radon as natural tracer - Quantification of groundwater inflow $\cdot$ Retention of iron precipitates on the catchment scale $\cdot$ Iron precipitation in the Spree river

\section{Einleitung}

Das Lausitzer Revier ist eins der größten noch aktiven Abbaugebiete für Braunkohle in Europa. Das Gebiet erstreckt sich vom Südosten Brandenburgs bis in den Nordosten Sachsens und liefert insgesamt rund ein Drittel der in Deutschland geförderten Braunkohle (Benthaus et al. 2015). Für die Braunkohleförderung wird der Grundwasserspiegel lokal um bis zu $100 \mathrm{~m}$ abgesenkt (Hüttl 1998). In seiner maximalen Ausdehnung erstreckte sich der dabei entstandene Absenktrichter insgesamt über eine Fläche von $2.100 \mathrm{~km}^{2}$ bis über die Grenzen des aktiven Tagebaus hinaus (Fleischhammel et al. 2010). Die tertiären und pleistozänen Bodenschichten in der Lausitz enthalten große Mengen an Eisendisulfid $\left(\mathrm{FeS}_{2 s}\right.$ ) wie Pyrit und Markasit (Meyer et al. 1999). Durch die Grundwasserabsenkung bedingte Belüftung des zuvor wassergesättigten und anoxischen Sedimentmaterials, kommt es zur Oxidation (Gl. 1-4) von $\mathrm{FeS}_{2 s}$ in den Abraumhalden sowie im Aquifer (Moses et al. 1987; Edwards et al. 1999; Schultze et al. 2010).

$$
\begin{aligned}
& \mathrm{FeS}_{2_{s}}+\frac{7}{2} \mathrm{O}_{2}+\mathrm{H}_{2} \mathrm{O} \rightarrow \mathrm{Fe}^{2+}+2 \mathrm{SO}_{4}^{2-}+2 \mathrm{H}^{+} \\
& \mathrm{Fe}^{2+}+\frac{1}{4} \mathrm{O}_{2}+\mathrm{H}^{+} \rightarrow \mathrm{Fe}^{3+}+\frac{1}{2} \mathrm{H}_{2} \mathrm{O} \\
& \mathrm{Fe}^{3+}+3 \mathrm{H}_{2} \mathrm{O} \rightarrow \mathrm{Fe}(\mathrm{OH})_{3_{s}}+3 \mathrm{H}^{+} \\
& \mathrm{FeS}_{2_{s}}+14 \mathrm{Fe}^{3+}+8 \mathrm{H}_{2} \mathrm{O} \rightarrow 15 \mathrm{Fe}^{2+}+2 \mathrm{SO}_{4}^{2-}+16 \mathrm{H}^{+}
\end{aligned}
$$

Kommt $\mathrm{FeS}_{2 s}$ mit Sauerstoff $\left(\mathrm{O}_{2}\right)$ in Kontakt wird es zu Sulfat $\left(\mathrm{SO}_{4}{ }^{2-}\right)$ oxidiert, wobei zweiwertiges gelöstes Eisen $\left(\mathrm{Fe}^{2+}\right)$ und Protonen $\left(\mathrm{H}^{+}\right)$freigesetzt werden (Gl. 1). Unter $\mathrm{O}_{2}$-Verfügbarkeit wird das gelöste $\mathrm{Fe}^{2+}$ weiter zu dreiwertigem Eisen $\left(\mathrm{Fe}^{3+}\right)$ oxidiert, welches wiederum durch Hydrolyse Eisenhydroxide $\left(\mathrm{Fe}(\mathrm{OH})_{3 s}\right)$ bildet und $\mathrm{H}^{+}$freisetzt (Gl. 3). Abhängig vom pH-Wert sowie der lokalen $\mathrm{O}_{2}$-Verfügbarkeit im Aquifer fungiert auch $\mathrm{Fe}^{3+}$ als Oxidationsmittel für $\mathrm{FeS}_{2 s}$ (Gl. 4). Dieser anoxische Prozess läuft unter erneuter Freisetzung von $\mathrm{Fe}^{2+}$ und $\mathrm{H}^{+}$ab. Durch Niederschlag und Sickerwasser, beim Wiederanstieg des Grundwassers und Flutung der Tagebaugruben, werden die Reaktionsprodukte (Gln. 1-4) gelöst und durch die Grundwasserbewegung in Richtung der Vorfluter (Kleine Spree und Spree), mobilisiert (Bilek et al. 2016). Beim Eintritt in die Fließgewässer bzw. bei direkter $O_{2}$-Verfügbarkeit im Aquifer fällt das schwer lösliche $\mathrm{Fe}(\mathrm{OH})_{3 s}$ unter $\mathrm{pH}$ neutralen Bedingungen in Form von Mikroflocken aus (Gleisner und Herbert
Jr 2002; Pham et al. 2006; Schultze et al. 2010), während $\mathrm{SO}_{4}{ }^{2-}$ im gelösten Zustand verbleibt. Ab einer Konzentration von 2-3 $\mathrm{mg} \mathrm{l}^{-1}$ im Fließgewässer wird das ausgefallene $\mathrm{Fe}(\mathrm{OH})_{3 s}$ durch rot-bräunliche Verfärbung der Suspension und des Sediments sichtbar (Ulrich et al. 2019).

Der in den Fließgewässern entstehende Eisenocker $\left(\mathrm{Fe}(\mathrm{OH})_{3 s}\right.$-Schlamm) wird je nach Fließeigenschaften des Vorfluters am Flussbett abgelagert. Dies hat negative Auswirkungen auf die benthische Flora und Fauna zur Folge, die durch die Eisenocker-Schlammschicht einen Mangel an Sauerstoff und Nährstoffen erfahren. Darüber hinaus kommt es zu einer Absenkung des lokalen pH-Wertes, was sich ebenfalls negativ auf aquatische Lebewesen auswirken kann. Ab einer $\mathrm{Fe}^{2+}$-Konzentrationen von $2-3 \mathrm{mgl}^{-1}$ in den Fließgewässern verenden Fischlaich, Invertebraten und Fische, letztere durch Verkleben der Kiemen (Kruspe et al. 2014). Die durch die starke Trübung erhöhte Lichtabsorption der Gewässer, führt zu einer verminderten Photosyntheserate, mit negativen Auswirkungen auf die gesamte Nahrungskette in fluvialen Ökosystemen (Kruspe et al. 2014). Neben den ökologischen Konsequenzen bringen die erhöhten Eisenkonzentrationen in den Fließgewässern auch wasserwirtschaftliche Probleme mit sich (Schwarzmüller und Menz 2013). Die Spree ist primäre Trinkwasserquelle für den Großraum Berlin, weshalb aufwändige Trinkwasseraufbereitungsmaßnahmen notwendig sind bzw. in Zukunft verstärkt notwendig werden.

Insbesondere der diffuse Eintrag von gelöstem $\mathrm{Fe}^{2+}$ durch das lokale Grundwasser könnte in der Lausitz eine zentrale Rolle bei der Verockerungsproblematik der Spree bzw. der Kleinen Spree spielen. Die Interaktion zwischen Fließgewässer und Grundwasser und der damit verbundene Austausch von Wasser und gelösten Stoffen, kann räumlich entlang eines Fließgewässers stark variieren (Frei et al. 2009). Ziel dieser Studie ist es 1) den Eintrag von Grundwasser entlang der Spree bzw. Kleinen Spree räumlich zu quantifizieren, 2) den Einfluss des Grundwassers auf den $\mathrm{Fe}^{2+}$-Eintrag in die Spree und Kleine Spree qualitativ und quantitativ zu verstehen sowie 3) die Bilanzierung der im Einzugsgebiet zurückgehaltenen Menge an ausgefallenem $\mathrm{Fe}(\mathrm{OH})_{3 s}$ aus der $\mathrm{FeS}_{2 s^{-}}$Verwitterung vorzunehmen. Die Lokalisierung und Quantifizierung der Grundwasserzuflüsse in die Spree und Kleine Spree erfolgt mithilfe des natürlichen Tracers Radon $\left({ }^{222} R n\right)$, einem geogen vorliegenden, radioaktiven Isotop der Uran-238-Zerfallsreihe. 
Abb. 1 Lage des Untersuchungsgebiets mit den beiden untersuchten Flüssen Kleine Spree (KS) und Spree (S) sowie den jeweiligen Probennahmepunkten

Fig. 1 Location of the study site including the two rivers Kleine Spree (KS) and Spree (S) with the corresponding sampling points

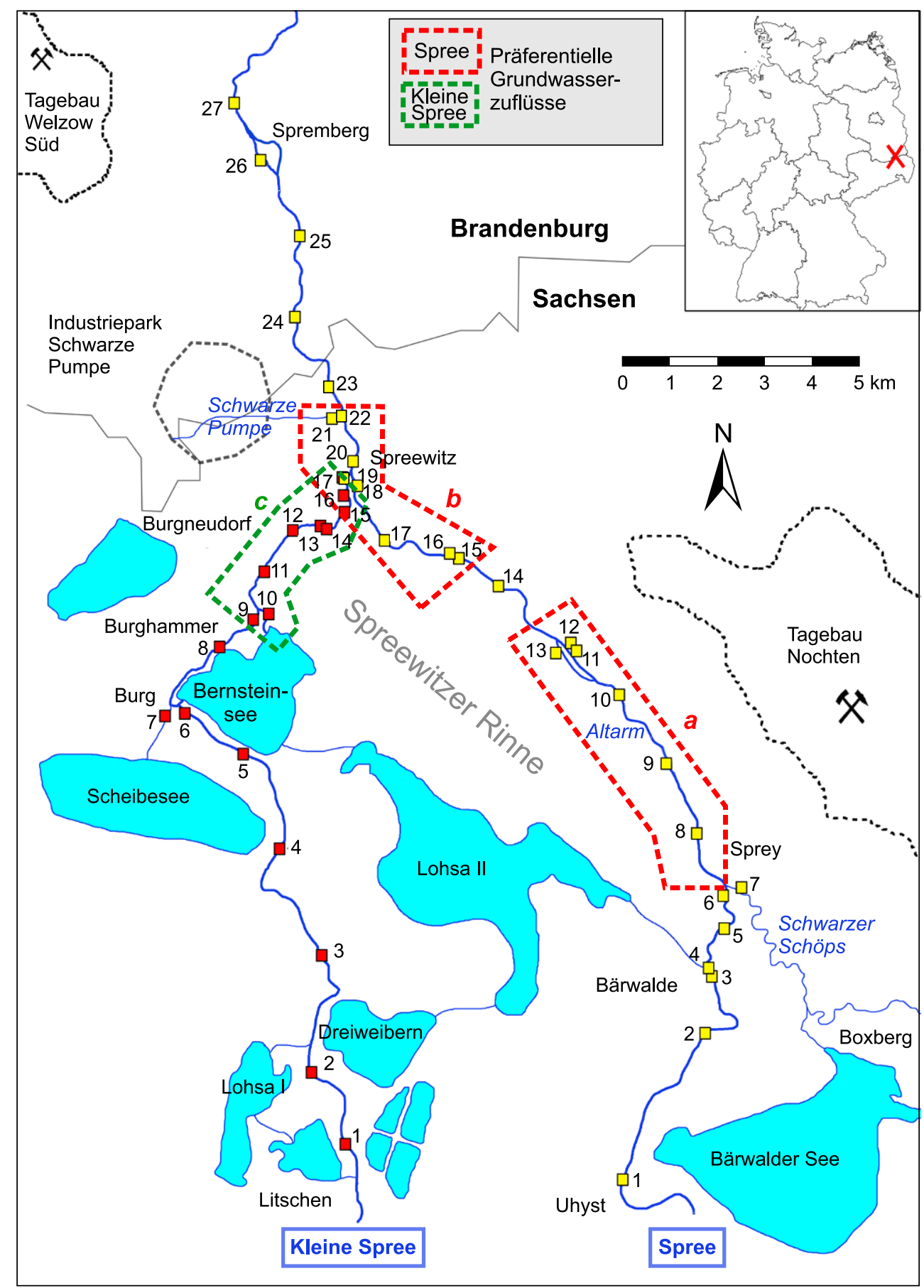

\section{Material und Methoden}

\section{Untersuchungsgebiet und Probennahme}

Die Probennahme entlang der Spree und der Kleinen Spree erfolgte während zwei Messkampagnen, zwischen dem 01.05. und dem 04.05.2018 sowie zwischen dem 13.08. und dem 16.08.2018. In beiden Messkampagnen wurden etwa $20 \mathrm{~km}$ Flussabschnitt der Kleinen Spree und $34 \mathrm{~km}$ der Spree in Abständen von $0,5-3 \mathrm{~km}$ beprobt (Abb. 1).
Ebenfalls wurden alle Hauptzuflüsse in die Kleine Spree bzw. Spree beprobt. Wichtige oberirdische Zuflüsse in die Kleine Spree sind der Scheibesee und der Bernsteinsee, und für die Spree der Zufluss Schwarzer Schöps/Bärwalder See, der Altarm am Wehr Ruhlmühle, die Kleine Spree und der Industriekanal Schwarze Pumpe. Wasserproben für ${ }^{222} \mathrm{Rn}, \mathrm{SO}_{4}^{2-}$ und gelöstem $\mathrm{Fe}^{2+}$ wurden mithilfe eines $11-$ Schöpfers in der Hauptströmung etwa $20-30 \mathrm{~cm}$ unter der Wasseroberfläche genommen. Für Flüsse mit sehr großen Fließbreiten (z. B. Rhein oder Donau) kann es zu schlechter 
horizontaler Durchmischung des durch das Grundwasser einströmende ${ }^{222} R n$ und anderer gelöster Stoffe kommen. Für Flüsse dieser Kategorie ist eine einzelne Probe oft nicht repräsentativ für den gesamten Fließquerschnitt. Bei der Spree bzw. Kleinen Spree in der Lausitz, mit einer maximalen Breite von bis $20 \mathrm{~m}$, gehen wir von einer guten vertikalen/horizontalen Durchmischung aus, weswegen jeweils nur eine Probe genommen wurde um den Fließquerschnitt an der entsprechenden Stelle zu repräsentieren. In der zweiten Messkampagne wurden zusätzlich 21 Grundwassermesstellen über das gesamte Untersuchungsgebiet verteilt beprobt (s. Karte in Abb. A1 im Anhang, zu finden im Zusatzmaterial der Online-Ausgabe diese Beitrags). Grundwasserproben für die Bestimmung der ${ }^{222} \mathrm{Rn}$-Aktivitäten wurden mithilfe einer Saugpumpe entnommen. Das abgepumpte Grundwasser wurde über den Schlauch der Pumpe in eine 101-PVC-Wanne geleitet und das Volumen je nach Tiefe und Durchmesser der Grundwasserbrunnen so lange ausgetauscht (nach Möglichkeit mindestens das 2-fache Volumen), bis sich repräsentative Grundwasserparameter (Temperatur, Leitfähigkeit, Sauerstoffgehalt) eingestellt hatten. Eine genaue Beschreibung der Probenaufbereitung der einzelnen Messparameter befindet sich im Anhang.

\section{Radon als natürlicher Tracer für die Quantifizierung von Grundwasserzuflüssen}

Das Edelgas Radon $\left({ }^{222} R n\right)$, mit einer Halbwertszeit von 3,8 Tagen, ist ein natürliches Zerfallsprodukt der Uran-BleiZerfallsreihe. Im Grundwasser stellt der radioaktive Zerfall des Mutterisotops Radium-226, enthalten in den mineralischen Bestandteilen des Aquifers, die einzige Quelle für ${ }^{222} R n$ dar. Senken für ${ }^{222} R n$ im Aquifer ist der radioaktive Zerfall und in Oberflächengewässern zusätzlich die Entgasung in die Atmosphäre (Cartwright und Gilfedder 2015). Im Grundwasser erreicht die ${ }^{222} R n$-Aktivität nach etwa 20 Tagen ( 5-fache Halbwertszeit) ein säkulares Gleichgewicht, bedingt durch den radioaktiven Zerfall sowie die Nachlieferungsrate von ${ }^{222} R n$ aus dem Zerfall des Mutterisotops ${ }^{226} \mathrm{Ra}$. Aufgrund der deutlichen Unterschiede in der ${ }^{222} R n$-Aktivität zwischen Oberflächen- und Grundwasser, eignet sich ${ }^{222} R n$ als natürlicher Tracer für die qualitative und quantitative Bestimmung von Grundwasserzuflüssen in Fließgewässer (Cook et al. 2006; Cook 2013; Gilfedder et al. 2015, 2019).

Die quantitative Auswertung der ${ }^{222} R n$-Messungen erfolgte mithilfe des Modelles FINIFLUX (Frei und Gilfedder 2015). FINIFLUX basiert auf einem Finite-Elemente-
Ansatz für die räumliche Diskretisierung der ${ }^{222} R n$-Massenbilanz in Fließgewässern (Gl. 5).

$$
\begin{aligned}
& Q_{s} \frac{d c}{d x}=I\left(c_{g w}-c\right)-k w c-d w \lambda \mathrm{c}+\frac{Q_{r}}{R_{L}}\left(c_{\text {trib }}-\mathrm{c}\right) \\
& +\frac{\gamma \mathrm{hw} \Theta}{1+\lambda t_{h}}-\frac{\lambda h w \Theta}{1+\lambda t_{h}} \cdot c
\end{aligned}
$$

Dabei bezeichnet $Q_{s}$ und $Q_{r}\left[\mathrm{~L}^{3} \mathrm{~T}^{-1}\right]$ den volumetrischen Abfluss im beprobten Flussabschnitt bzw. in den Zuflüssen, $x[\mathrm{~L}]$ die Länge des Flussabschnittes, $I\left[\mathrm{~L}^{3} \mathrm{~T}^{-1} \mathrm{~L}^{-1}\right]$ die lokale Grundwasserzuflussrate, $c, c_{g w}$ und $c_{\text {trib }}\left[\mathrm{M} \mathrm{L}^{-3}\right]$ die ${ }^{222} \mathrm{Rn}$-Aktivitäten im Fluss, im Grundwasser sowie in $\mathrm{Zu}$ flüssen, $k\left[\mathrm{~T}^{-1}\right]$ den ${ }^{222} R n$ Entgasungskoeffizienten, $w[\mathrm{~L}]$ die Flussbreite, $d[\mathrm{~L}]$ die Flusstiefe und $\lambda\left[\mathrm{T}^{-1}\right]$ die radioaktive Zerfallskonstante von ${ }^{222} R n$.

Die Anreicherung von ${ }^{222} R n$ in den Flussbettsedimenten durch den hyporheischen Austausch wird durch die letzten beiden Terme in Gl. 5 beschrieben. Hier steht $\gamma$ $\left[\mathrm{M} \mathrm{L}^{-3} \mathrm{~T}^{-1}\right]$ für die ${ }^{222} R n$-Produktionsrate im Flussbettsediment, $h[\mathrm{~L}]$ für die Tiefe des hyporheischen Austausches, $\Theta[-]$ für die Porosität der Flussbettsedimente und $t_{h}[\mathrm{~T}]$ für die mittlere Verweilzeit des infiltrierten Flusswassers in der hyporheischen Zone, basierend auf einer exponentiellen Verweilzeitverteilung. FINIFLUX ist gekoppelt an den Optimierungsalgorithmus PEST (Doherty et al. 1994), für die inverse Bestimmung sowohl der Grundwasserzuflussraten $I$ als auch der hyporheischen Parameter $t_{h}$ und $h$. Die ${ }^{222} R n$-Aktivität des Grundwassers $\left(c_{g w}\right.$ in Gl. 5) stellt mitunter die größte Unsicherheit bei der inversen Bestimmung der Grundwasserzuflussraten als Teil der FINIFLUXModellierung dar. Auf Basis der im Untersuchungsgebiet gemessenen ${ }^{222} R n$-Aktivitäten der Grundwassermesstellen (siehe Anhang) wurde eine Häufigkeitsverteilung der Endmember-Aktivitäten erstellt. Für die Häufigkeitsverteilung wurde angenommen, dass die gemessene Variabilität in den ${ }^{222} R n$-Aktivitäten der Grundwasserproben einer Normalverteilung folgt. Anschließend wurde für die normalverteilte Grundgesamtheit das 25\%-, 50\%- und 75\%-Quantil berechnet. Die FINIFLUX-Simulationen wurden jeweils mit allen drei Quantilen, als entsprechende Endmember-Konzentration $\left(c_{g w}\right.$ in Gl. 5) durchgeführt, um so den Einfluss der Unsicherheit auf die ermittelten Grundwasserzuflussraten zu bestimmen. Die Abflussdaten bzgl. der Spree und der Kleinen Spree sowie aller Zuflüsse wurden für beide Messkampagnen von der Lausitzer und Mitteldeutschen Bergbau-Verwaltungsgesellschaft (LMBV), vom Landesamt für Umwelt Brandenburg (LfU), vom Landesamt für Umwelt, Landwirtschaft und Geologie Sachsen (LfULG) und von der Landestalsperrenverwaltung Sachsen (LTV) bereitgestellt und sind im Anhang hinterlegt. 


\section{Bestimmung der im Einzugsgebiet der Spree zurückgehaltenen Menge an Eisen aus der Eisendisulfidoxidation}

Als Teil dieser Studie wird angenommen, dass die verschiedenen Eisen- und Sulfat-Verbindungen in der Spree und der Kleinen Spree ursprünglich aus der Oxidation von $\mathrm{FeS}_{25^{-}}$ Mineralen stammen. Unter der Annahme, dass $\mathrm{SO}_{4}{ }^{2-}$ im Gegensatz zu gelöstem $\mathrm{Fe}^{2+}$ chemisch inerte Eigenschaften im Einzugsgebiet (Tagebaurestseen, Fließgewässer und Aquifer) aufweist, kann über die gemessenen $\mathrm{SO}_{4}{ }^{2-}$-Konzentration $c\left(\mathrm{SO}_{4}{ }^{2-}\right)\left[\mathrm{M} \mathrm{l}^{-3}\right]$ die Soll-Konzentration von Eisen $c\left(\mathrm{Fe}_{\text {soll }}\right)$ $\left[\mathrm{M} \mathrm{L}^{-3}\right]$ in den Fließgewässern ermittelt werden (Gl. 6). Der stöchiometrische Faktor 0,5 in Gl. 6 indiziert dabei, dass aus der Oxidation von $\mathrm{FeS}_{2 s}$ jeweils nur halb so viel $\mathrm{Fe}^{2+}$ wie $\mathrm{SO}_{4}{ }^{2-}$ gebildet wird. Die Soll-Konzentration an gelöstem $\mathrm{Fe}^{2+} c\left(\mathrm{Fe}_{\text {soll }}\right)$ entspricht somit der hypothetischen Konzentration in den Vorflutern für den Fall, dass gelöstes $\mathrm{Fe}^{2+}$ im Einzugsgebiet konservativ transportiert wird. Über die in den Fließgewässern gemessene tatsächliche $\mathrm{Fe}^{2+}$-Konzentration $c\left(F e_{i s t}\right)\left[\mathrm{M} \mathrm{L}^{-3}\right]$ sowie dem gemessenen Abfluss $Q s$, kann über Gl. 7 die im Einzugsgebiet zurückgehaltene, immobilisierte Menge an Eisen $\left[\mathrm{Fe}_{i m m}\right]\left[\mathrm{M} \mathrm{T}^{-1}\right]$ ermittelt werden. Dabei entspricht $\left[\mathrm{Fe}_{\text {soll }}\right]\left[\mathrm{M} \mathrm{T}^{-1}\right]$ der Soll-Fracht an gelöstem $\mathrm{Fe}^{2+}$ und $\left[\mathrm{Fe}_{i s t}\right]\left[\mathrm{M} \mathrm{T}^{-1}\right]$ der tatsächlich gemessenen Fracht in den Fließgewässern. Die für Gl. 7 notwendigen Konzentrationen $c\left(\mathrm{SO}_{4}{ }^{2-}\right)$ und $c\left(\mathrm{Fe}_{i s t}\right)$ wurden für die untersuchten Flussabschnitte ionenchromatographisch $\left(c\left(\mathrm{SO}_{4}^{2-}\right)\right)$ bzw. photometrisch $\left(c\left(F e_{i s t}\right)\right)$ bestimmt (nähere Informationen zur Probennahme und Analyse befinden sich im Anhang).

$$
\begin{aligned}
& c\left(F e_{\text {soll }}\right)=\frac{1}{2} c\left(S O_{4}^{2-}\right) \\
& {\left[F e_{\text {imm }}\right]=\left[F e_{\text {soll }}\right]-\left[F e_{\text {ist }}\right]=Q s\left[c\left(F e_{\text {soll }}\right)-c\left(F e_{\text {ist }}\right)\right]}
\end{aligned}
$$

\section{Ergebnisse}

\section{Rn-Aktivitäten in der Spree und der Kleinen Spree}

Im Mittel lagen die gemessenen ${ }^{222} \mathrm{Rn}$-Aktivitäten in der Kleinen Spree für Messkampagne $1\left(90 \mathrm{~Bq} \mathrm{~m}^{-3}\right)$ über den Werten der Messkampagne $2\left(47 \mathrm{~Bq} \mathrm{~m}^{-3}\right)$ (Abb. 2). Die ${ }^{222}$ Rn-Aktivitäten zu Beginn der beprobten Fließstrecke der Kleinen Spree lagen bei $184 \mathrm{~Bq} \mathrm{~m}^{-3}$ (Messkampagne 1) und $59 \mathrm{~Bq} \mathrm{~m}{ }^{-3}$ (Messkampagne 2), mit jeweils abfallendem Trend über die ersten $10 \mathrm{~km}$. Die ${ }^{222} \mathrm{Rn}$-Aktivitäten des Zuflusses durch den Scheibesee lagen für beide Messkampagnen unterhalb der Nachweisgrenze $\left(<15 \mathrm{~Bq} \mathrm{~m}^{-3}\right)$. Durch den Zufluss von nahezu ${ }^{222} \mathrm{Rn}$-freien Wassers aus dem Scheibesee kommt es in der Kleinen Spree zu einem Verdünnungseffekt, bei dem die gemessene ${ }^{222} \mathrm{Rn}$-Aktivität zwischen den Messpunkten 6 und 7 (Abb. 2) deutlich abfällt. Im Bernsteinsee lagen die gemessenen ${ }^{222} \mathrm{Rn}$-Aktiviäten während Messkampagne 1 mit $68 \mathrm{~Bq} \mathrm{~m}^{-3}$ nah an den Aktivitäten in der Kleinen Spree (Messpunkt 9, Abb. 2). Für Messkampagne 2 lag die ${ }^{222} \mathrm{Rn}$-Akvität im Bernsteinsee $\left(62 \mathrm{~Bq} \mathrm{~m}^{-3}\right)$ über der gemessenen Aktivität der Kleinen Spree $\left(\sim 20 \mathrm{~Bq} \mathrm{~m}^{-3}\right)$, was einen sprunghaften Anstieg der ${ }^{222} \mathrm{Rn}$-Akvität im Zuflussbereich zwischen den Messpunkten 9 und 10 verursachte. Für die untersuchten Flussabschnitte, im Bereich des Zusammenflusses mit der Spree, zeigte sich für beide Messkampagnen eine tendenzielle $\mathrm{Zu}$ nahme der ${ }^{222}$ Rn-Aktivitäten.

Wie bereits für die Kleine Spree festgestellt, waren die ${ }^{222} \mathrm{Rn}$-Aktivitäten im Mittel für die Spree während Messkampagne $1\left(267 \mathrm{~Bq} \mathrm{~m}^{-3}\right)$ höher als für Messkampagne 2 (215 Bq m${ }^{-3}$ ) (Abb. 2). Im Vergleich zur Kleinen Spree lagen die gemessenen Werte der Spree deutlich über den Werten der Kleinen Spree, was auf einen höheren Grundwassereinfluss hindeutet. Zu Beginn der untersuchten Fließstrecke lagen die ${ }^{222} \mathrm{Rn}$-Aktivitäten in der Spree bei $522 \mathrm{~Bq} \mathrm{~m}^{-3}$ (Messkampagne 1) bzw. $271 \mathrm{~Bq} \mathrm{~m}^{-3}$ (Messkampagne 2). Für die ersten $5 \mathrm{~km}$ der untersuchten Fließstrecke sanken die gemessenen ${ }^{222} \mathrm{Rn}$-Aktivitäten deutlich. Zwischen den Messpunkten 3 und 4 kam es aufgrund einer wehrbedingten Entgasung zu einer deutlichen Reduktion der ${ }^{222} \mathrm{Rn}$ Aktivitäten von 390 auf $143 \mathrm{~Bq} \mathrm{~m}^{-3}$ (Messkampagne 1) bzw. von 280 auf $119 \mathrm{~Bq} \mathrm{~m}^{-3}$ (Messkampagne 2). Für beide Messkampagnen lagen die ${ }^{222} \mathrm{Rn}$-Aktivitäten des Zuflusses Schwarzer Schöps ( 430 Bq m${ }^{-3}$ für beide Messkampagnen) deutlich über den Aktivitäten in der Spree. Hier kam es im Bereich des Zuflusses zwischen den Messpunkten 6 und 7 zu einer deutlichen Zunahme der ${ }^{222} \mathrm{Rn}$-Aktivitäten in der Spree. Die mit Abstand höchsten gemessenen ${ }^{222}$ Rn-Aktivitäten $\left(>1000 \mathrm{~Bq} \mathrm{~m}^{-3}\right)$ wurden für den Altarm der Spree zwischen den Messpunkten 12 und 13 gemessen. Aufgrund der hohen ${ }^{222} \mathrm{Rn}$-Aktivitäten des Altarms (Messkampagne 1: $1373 \mathrm{~Bq} \mathrm{~m}^{-3}$ und Messkampagne 2: $1532 \mathrm{~Bq} \mathrm{~m}^{-3}$ ) kam es in diesem Bereich zu einer lokalen Erhöhung der ${ }^{222} \mathrm{Rn}$ Aktivitäten in der Spree. Der Anstieg der ${ }^{222}$ Rn-Aktivitäten, verursacht durch den Zufluss des Altarms, ist im Vergleich zu anderen Zuflüssen (z.B. Schwarzer Schöps) nur sehr moderat ausgeprägt, da der Spree durch den Altarm nur geringe Mengen an Wasser zufließen. Nach $22 \mathrm{~km}$ folgten die ${ }^{222} \mathrm{Rn}$-Aktivitäten einem Abwärtstrend, bis Werte von $174 \mathrm{~Bq} \mathrm{~m}^{-3}$ (Messkampagne 1) und $137 \mathrm{~Bq} \mathrm{~m}^{-3}$ (Messkampagne 2) am Ende des untersuchten Fließabschnittes erreicht wurden. Die ${ }^{222} \mathrm{Rn}$-Aktivitäten der Zuflüsse Kleine Spree nach $22 \mathrm{~km}$ mit $103 \mathrm{~Bq} \mathrm{~m}^{-3}$ (Messkampagne 1) bzw. $51 \mathrm{~Bq} \mathrm{~m}^{-3}$ (Messkampagne 2) und Schwarze Pumpe nach

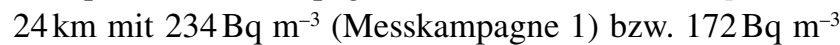
(Messkampagne 2) führten zu einem leichten Verdünnungseffekt der ${ }^{222} \mathrm{Rn}$-Aktivitäten in der Spree. 

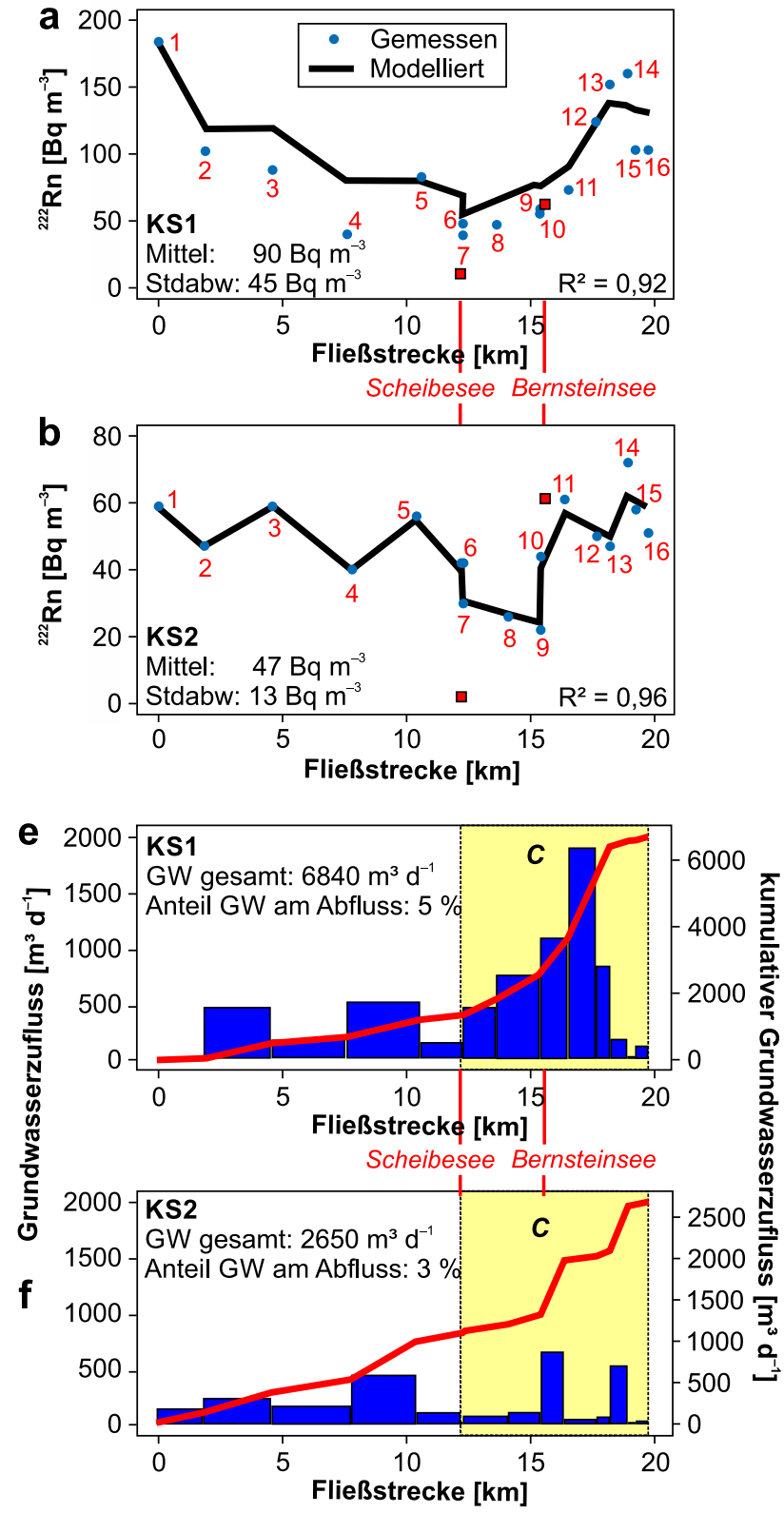

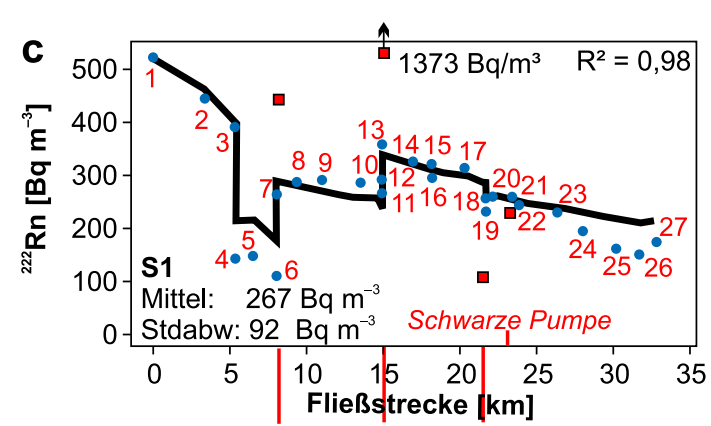

Schwarzer Schöps Altarm Kleine Spree
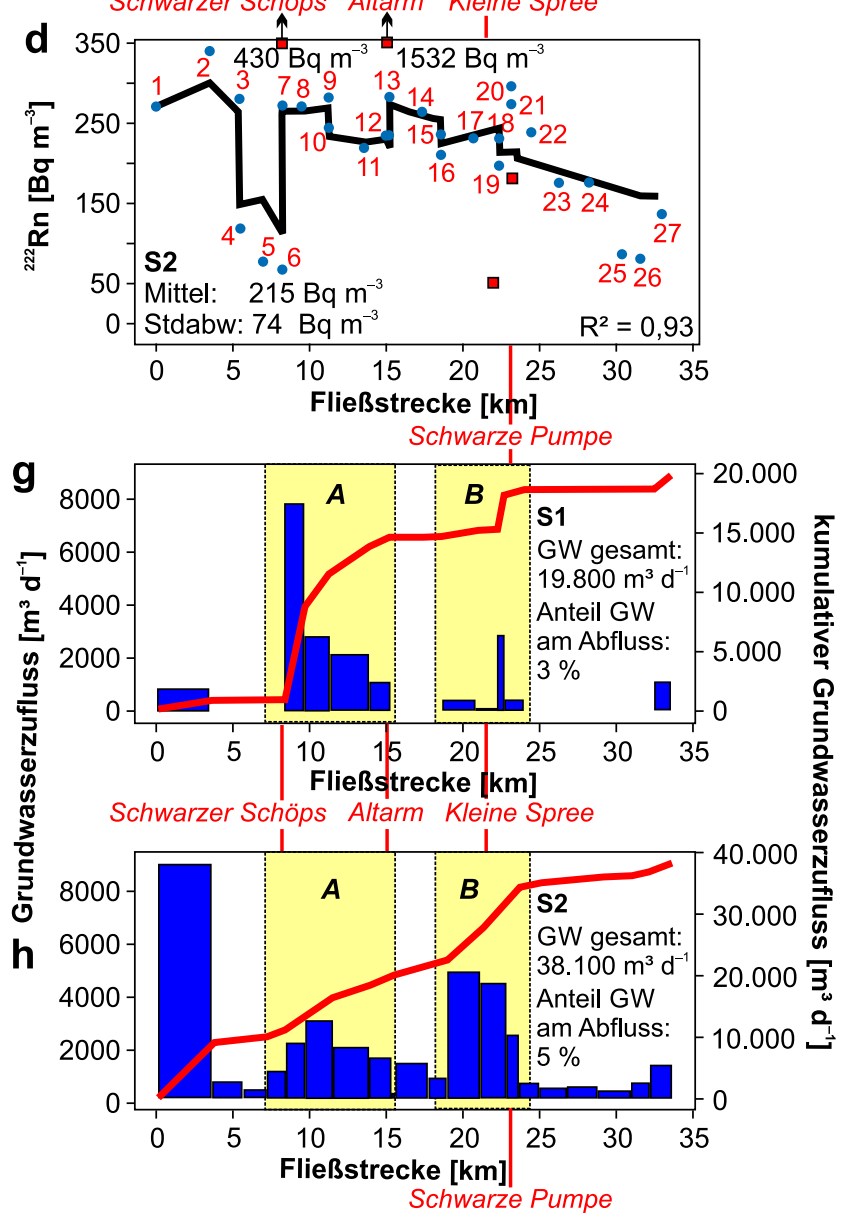

Abb. 2 Gemessene und modellierte ${ }^{222}$ Rn-Konzentrationen für die Spree (S) und die Kleine Spree (KS) für Messkampagne 1 und 2 (a-d) sowie simulierter Grundwasserzufluss für die untersuchten Flussabschnitte der Spree und der Kleinen Spree für beide Messkampagnen (e-h)

Fig. 2 Observed and simulated ${ }^{222} \mathrm{Rn}$-activities for the Spree (S) and Kleine Spree (KS) for both measurement campaigns (a-d), and simulated groundwater inflow for the different river reaches of the Spree and Kleine Spree $(\mathbf{e}-\mathbf{h})$

\section{Quantifizierung des lokalen Grundwasserzuflusses in die Spree und in die Kleine Spree}

Für beide Messkampagnen wurden die lokalen Grundwasserzuflussraten, basierend auf den gemessenen EndmemberAktivitäten der Grundwasserproben sowie der ${ }^{222}$ Rn-Aktivitäten in der Spree und der Kleinen Spree, durch FINIFLUX bestimmt. Die ${ }^{222} \mathrm{Rn}$-Aktivitäten der Grundwasserproben lagen für die einzelnen Messstellen ( $n=18$, Karte siehe An- hang) zwischen 1410 und $7840 \mathrm{~Bq} \mathrm{~m}^{-3}$. Der Median, der als Endmember-Aktivität für die Simulationen in Abb. 2 benutzt wurde ( $c_{g w}$ in Gl. 5), lag bei $2565 \mathrm{~Bq} \mathrm{~m}^{-3}$ und die beiden $25 \%$ - und $75 \%$-Quantile bei 1852 und $3185 \mathrm{~Bq} \mathrm{~m}^{-3}$. Sowohl für die Spree als auch die Kleine Spree zeigen die Simulationen eine gute Übereinstimmung zwischen den modellierten und gemessenen ${ }^{222} \mathrm{Rn}$-Aktivitäten $\left(\mathrm{R}^{2}>90 \%\right)$.

Über die gesamte untersuchte Fließstrecke der Kleinen Spree liegt der gesamte, kumulierte Grundwasserzustrom 


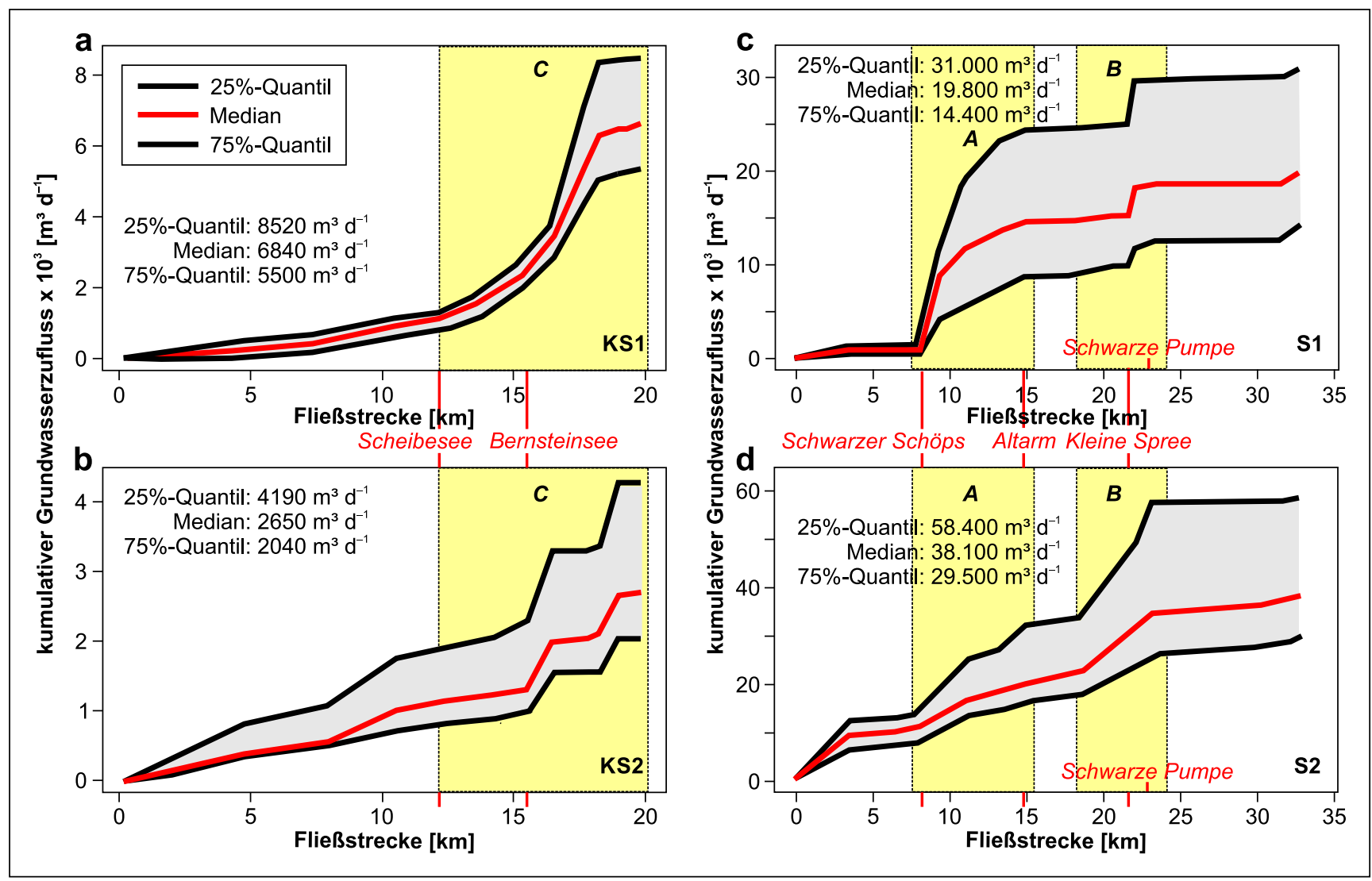

Abb. 3 Abschätzung des Einflusses der Unsicherheit der Grundwasser-Endmember-Aktivität auf die Quantifizierung der Grundwasserzuflussraten für die Spree und Kleine Spree. Simulationen wurden durchgeführt unter Verwendung des 25\%- und 75\%-Quantils sowie des Medians der gemessenen ${ }^{222} \mathrm{Rn}$-Grundwasseraktiviäten. Die Bereiche A-C kennzeichnen Flussabschnitte mit präferentiellem Grundwassereintritt

Fig. 3 Calculated uncertainty bands for groundwater inflow to the Spree and Kleine Spree based on the 25\%, 50\% (median) and $75 \%$ quantiles of the observed ${ }^{222} \mathrm{Rn}$ groundwater activity. Yellow areas represent areas of preferential groundwater inflow

bei $\sim 6900 \mathrm{~m}^{3} \mathrm{~d}^{-1}$ für Messkampagne 1 und bei $\sim 2700 \mathrm{~m}^{3} \mathrm{~d}^{-1}$ für Messkampagne 2. Im Mittel waren die simulierten Grundwasserzuflussraten für Messkampagne $1\left(456 \mathrm{~m}^{3} \mathrm{~d}^{-1}\right)$ deutlich höher als für Messkampagne $2\left(176 \mathrm{~m}^{3} \mathrm{~d}^{-1}\right)$. Basierend auf den durchgeführten Simulationen zeigt die Kleine Spree im Mündungsbereich zur Spree entlang der Spreewitzer Rinne (Bereich C in Abb. 1 und 2) während Messkampagne 1 lokal erhöhte Grundwasserzuflussraten (zwischen 100 und $2000 \mathrm{~m}^{3} \mathrm{~d}^{-1}$ ). Für diesen Bereich liegt der Anteil am Gesamtzustrom bei $75 \%\left(\sim 5000 \mathrm{~m}^{3} \mathrm{~d}^{-1}\right)$. Der erhöhte Grundwasserzustrom im Mündungsbereich zur Spree erklärt die in diesem Abschnitt gemessenen erhöhten ${ }^{222} \mathrm{Rn}$-Aktivitäten. Für Messkampagne 2 liegt der Anteil am Gesamtzustrom im Mündungsbereich bei nur noch $58 \%$ $\left(\sim 1500 \mathrm{~m}^{3} \mathrm{~d}^{-1}\right)$.

Der kumulierte Grundwasserzustrom entlang der untersuchten Fließstrecke der Spree liegt für Messkampgane 1 bei $\sim 20.000 \mathrm{~m}^{3} \mathrm{~d}^{-1}$ und für Messkampagne 2 bei $\sim 38.000 \mathrm{~m}^{3} \mathrm{~d}^{-1}$. Der simulierte mittlere Grundwasserzufluss für die einzelnen Flussabschnitte der Spree lag während Messkampagne $1\left(733 \mathrm{~m}^{3} \mathrm{~d}^{-1}\right)$ deutlich unter den Wert für
Messkampagne $2\left(1412 \mathrm{~m}^{3} \mathrm{~d}^{-1}\right)$. Für die Spree wurden für beide Messkampagnen zwei Bereiche mit präferenziellem Grundwassereintritt identifiziert. Der erste Bereich (Bereich A in Abb. 1 und 2) liegt zwischen den Messpunkten 8 und 14 in dem Flussabschnitt entlang der Spreewitzer Rinne bis zur Einmündung des Altarms. Für Bereich A liegt der Anteil am gesamten Grundwasserzustrom bei $70 \%\left(\sim 13.700 \mathrm{~m}^{3} \mathrm{~d}^{-1}\right)$ für Messkampagne 1 und bei $23 \%$ $\left(\sim 9000 \mathrm{~m}^{3} \mathrm{~d}^{-1}\right)$ für Messkampagne 2. Der zweite Bereich des präferenziellen Grundwassereintritts in die Spree (Bereich $\mathrm{B}$ in Abb. 2) liegt weiter flussabwärts, ebenfalls entlang der Spreewitzer Rinne, zwischen den Messpunkten 15 und 22. Für Messkampagne 1 lag hier der simulierte Anteil des Grundwasserzuflusses im Bereich B bei $17 \%\left(\sim 3400 \mathrm{~m}^{3} \mathrm{~d}^{-1}\right)$ und für Messkampagne 2 bei $25 \%$ $\left(9400 \mathrm{~m}^{3} \mathrm{~d}^{-1}\right)$. Der höchste simulierte Grundwasserzustrom mit $>9000 \mathrm{~m}^{3} \mathrm{~d}^{-1}$ wurde für den ersten untersuchten Flussabschnitt der Spree, zwischen den Messpunkten 1 und 2 für Messkampagne 2 ermittelt (Abb. 2). Dieser hohe Grundwasserzustrom konnte jedoch durch die Simulationen für 


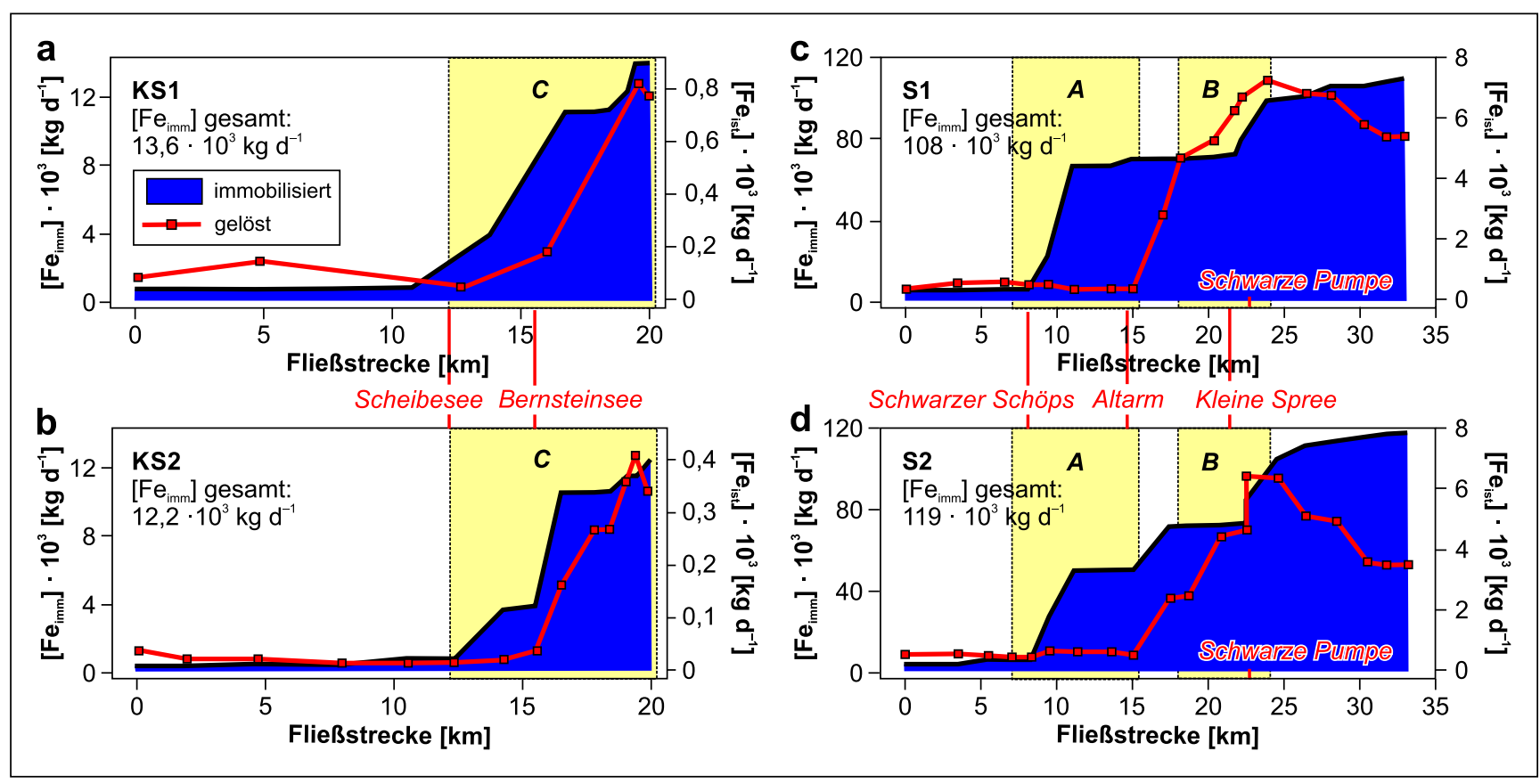

Abb. 4 Verlauf der gelösten Eisenfracht und der zurückgehaltenen Menge an Eisen in dem Einzugsgebiet der untersuchten Flussabschnitte der Kleinen Spree (KS) und der Spree (S)

Fig. 4 Dissolved iron load in the Spree and Kleine Spree and retained amount of iron in the catchment

Messkampagne 1 nicht bestätigt werden und wurde deshalb nicht als präferenzielle Grundwassereintrittszone deklariert.

Die Simulationen für die Quantifizierung des lokalen Grundwasserzuflusses in die Spree bzw. die Kleine Spree wurden unter Verwendung der $25 \%$ - und $75 \%$-Quantile der Grundwasser-Endmember-Aktivität $c_{g w}$ wiederholt (Abb. 3). Die kumulierten Grundwasserzuflussraten für das $75 \%$-Quantil liegen unter den Werten des $25 \%$-Quantils, da aufgrund der höheren Endmember-Aktivität (75\%-Quantil) weniger Grundwasser notwendig ist, um die in den Fließgewässern gemessenen ${ }^{222} \mathrm{Rn}$-Aktivitäten zu erklären. Zwar unterscheiden sich die absoluten Werte des Grundwasserzuflusses deutlich für die drei unterschiedlichen Szenarien, die zuvor identifizierten Bereiche präferentiellen Grundwassereintritts (Bereich A-C) bleiben jedoch auch unter Verwendung der $25 \%$ - und $75 \%$-Quantile erhalten.

\section{Im Einzugsgebiet zurückgehaltenen Menge an Eisen aus der Sulfidoxidation}

Für beide Fließgewässer korrelieren die gemessenen gelösten $\mathrm{Fe}^{2+}$-Konzentrationen $c\left(\mathrm{Fe}_{i s t}\right)$ mit den Bereichen präferenziellen Grundwassereintrittes. Hohe $\mathrm{Fe}^{2+}$-Konzentrationen wurden insbesondere für die Bereiche $\mathrm{C}$ und $\mathrm{B}$ entlang der Spreewitzer Rinne gemessen. Für die Fracht an gelöstem $\mathrm{Fe}^{2+}\left[\mathrm{Fe}_{i s t}\right]$ zeigte sich ein ähnlicher Verlauf entlang der Spree und der Kleinen Spree (Abb. 4). Für die oberirdischen Zuflüsse aus dem Scheibesee, Bernsteinsee sowie dem Bär- walder See wurden nur geringe gelöste $\mathrm{Fe}^{2+}$-Konzentrationen gemessen $\left(9-16 \mu \mathrm{moll}^{-1}\right)$. Die höchsten Konzentrationen für gelöstes $\mathrm{Fe}^{2+}\left(3,5\right.$ bzw. $\left.3,4 \mathrm{mmoll}^{-1}\right)$ und $\mathrm{SO}_{4}{ }^{2-}(7,1$ bzw. 7,3 $\mathrm{mmoll}^{-1}$ ) wurde im Altarm der Spree gemessen. Die hohen $\mathrm{Fe}^{2+}$ - und $\mathrm{SO}_{4}{ }^{2-}$-Konzentrationen, in Verbindung mit den hohen gemessenen ${ }^{222} R n$-Aktivitäten $\left(>1000 \mathrm{~Bq} \mathrm{~m}^{3}\right)$ in diesem Bereich, lassen darauf schließen, dass ein Großteil des Altarm-Wassers aus dem Grundwasser stammt. Für den Altarm liegt das Verhältnis aus gemessener $\mathrm{Fe}^{2+}$ - und $\mathrm{SO}_{4}^{2-}$-Konzentration sehr nahe $(\sim 97 \%)$ an dem stöchiometrischen Verhältnis aus Gl. 6, was darauf hinweist, dass nur geringe Mengen an $\mathrm{Fe}^{2+}$ im Altarm als $\mathrm{Fe}(\mathrm{OH})_{3 s}$ ausgefallen sind. Die hohen $\mathrm{Fe}^{2+}-$ Konzentrationen lassen sich durch die niedrigen gemessenen $\mathrm{pH}-$ Werte im Altarm (pH 5) sowie im vorfluternahen Grundwasser (pH 3-4) erklären.

Sowohl für die Spree als auch die Kleine Spree ist die zurückgehaltene Menge an ausgefallenem $\mathrm{Fe}(\mathrm{OH})_{3 s}\left[\mathrm{Fe}_{\text {imm }}\right]$ während der ersten untersuchten Flusskilometer vergleichsweise gering (Abb. 4). Für diese Bereiche liegen die Werte für die ermittelten $\mathrm{Fe}^{2+}$-Soll $\left[\mathrm{Fe}_{\text {soll }}\right]$ und Ist-Frachten $\left[\mathrm{Fe}_{i s t}\right]$ (Gl. 7) nahe beieinander. Eine starke Zunahme von $\left[\mathrm{Fe}_{i m m}\right]$ ergab sich für die Bereiche präferenziellen Grundwassereintrittes entlang der Spreewitzer Rinne (Bereich A-C, Abb. 4). Für diese Flussabschnitte ergab sich eine hohe Differenz zwischen $\left[F e_{\text {soll }}\right]$ und $\left[F e_{i s t}\right]$, was darauf hinweist, dass hier große Mengen an $\mathrm{Fe}^{2+}$ in Form von ausgefallenem $\mathrm{Fe}(\mathrm{OH})_{3 s}$ in den Flussabschnitten bzw. deren Einzugsgebieten zurückgehalten wurden. Im Bereich der oberirdischen Zuflüs- 
se in die Spree bzw. die Kleine Spree kommt es oft zu einem deutlichen Anstieg von $\left[F_{i m m}\right]$. Mit Ausnahme des Altarms weisen alle anderen Zuflüsse relativ hohe $\mathrm{SO}_{4}{ }^{2-}-\left(c\left(\mathrm{SO}_{4}{ }^{2-}\right)\right)$ und geringe $\mathrm{Fe}^{2+}$-Konzentrationen $\left(c\left(F e_{i s t}\right)\right)$ auf, was nach Gl. 7 die Zunahme von $\left[\mathrm{Fe}_{i m m}\right]$ in diesen Abschnitten erklärt (Abb. 4). Die hohe Differenz aus gemessener $\mathrm{Fe}^{2+}$ - und $\mathrm{SO}_{4}{ }^{2-}$-Konzentration in den Zuflüssen, lassen den Schluss $\mathrm{zu}$, dass in den Tagebaurestseen große Mengen an $\mathrm{Fe}^{2+} \mathrm{zu}-$ rückgehalten werden. Für das Einzugsbiet der untersuchten Flussabschnitte der Kleinen Spree ergibt sich rechnerisch eine zurückgehaltene Menge an Eisen von 14 (Messkampagne 1) bzw. 12 Tonnen/Tag (Messkampagne 2) und für die Spree von 108 (Messkampagne 1) und 119 Tonnen/Tag (Messkampagne 2).

\section{Diskussion}

Das durchgeführte ${ }^{222} \mathrm{Rn}$-Monitoring für die Spree und die Kleine Spree sowie die Ergebnisse der FINIFLUX-Simulationen zeigen, dass die Grundwasserzuflussraten für die einzelnen Flussabschnitte stark variieren und Bereiche mit präferenziellem Grundwasserzufluss existieren. Sowohl für die Spree als auch die Kleine Spree konnten, entlang der Spreewitzer Rinne, Bereiche mit präferenziellem Grundwassereintritt durch das ${ }^{222} \mathrm{Rn}$-Monitoring identifiziert werden. Im Bereich der Spreewitzer Rinne erfolgte um das Jahr 2000 eine Umkehr der Grundwasserfließrichtung von den Tagebaugruben (v. a. Lohsa II) zur Spree, was nachweislich zu verstärken Grundwasserzuflüssen und erhöhten Eiseneinträgen in die Spree führte (Uhlmann et al. 2012). Für die Flussabschnitte entlang der Spreewitzer Rinne liegt der Anteil am gesamten Grundwasserzustrom für die Kleine Spree bei $>50 \%\left(\sim 5000 \mathrm{~m}^{3} \mathrm{~d}^{-1}\right)$ für Messkampagne 1 und $>70 \%$ $\left(\sim 1500 \mathrm{~m}^{3} \mathrm{~d}^{-1}\right)$ sowie für Messkampagne 2 und für die Spree bei $>40 \%\left(\sim 17.000 \mathrm{~m}^{3} \mathrm{~d}^{-1}\right)$ bzw. $>70 \%\left(\sim 23.000 \mathrm{~m}^{3} \mathrm{~d}^{-1}\right)$. Für die Spree wurde von ähnlichen Raten für den Grundwasserzustrom (17.280-34.560 $\left.\mathrm{m}^{3} \mathrm{~d}^{-1}\right)$ entlang der Spreewitzer Rinne berichtet (Benthaus et al. 2015). Für die Kleine Spree weichen die Werte von Benthaus et al. (2015) (17.280-25.920 $\mathrm{m}^{3} \mathrm{~d}^{-1}$ ) allerdings deutlich von Raten $\mathrm{ab}$, die durch das ${ }^{222} \mathrm{Rn}$-Verfahren ermittelt wurden.

Rechts der Spree entlang der Spreewitzer Rinne zwischen Boxberg und Neustadt, befindet sich der wenige Kilometer entfernte, noch aktive Tagebau Nochten. Aufgrund der massiven Grundwasserabsenkung im Tagbau Nochten und des dadurch entstandenen Absenktrichters, wäre es möglich, dass die Flussabschnitte der Spree entlang der Spreewitzer Rinne sogenannte Durchflusssysteme darstellen. Durchflusssysteme können sich bei Oberflächengewässern einstellen und bezeichnen die Situation, dass eine Seite des Ufers Wasser aus dem Aquifer erhält, während die andere Wasser an den Aquifer abgibt (Nield et al. 1994; Woess- ner 2000). Trotz der simulierten positiven Grundwasserzuflussraten (effluente Bedingungen) in die Spree/Kleine Spree, kann nicht ausgeschlossen werden, dass lokale Fließabschnitte existieren, die Wasser an den Aquifer abgeben (influente Bedingungen). Influente Bedingungen lassen sich allerdings durch das verwendete ${ }^{222} \mathrm{Rn}$-Verfahren nicht bestimmen, da sich hier die ${ }^{222}$ Rn-Aktivität im Fließgewässer nicht ändert.

Die Fehler in der Wasserbilanz für die FINIFLUX-Simulationen lagen, mit Ausnahme für Messkampane 2 bei der Kleinen Spree (Fehler: 20\%), unter 5\% (siehe Anhang). Geringe Fehlerwerte stehen für eine geschlossene Wasserbilanz, was die Plausibilität der berechneten Grundwasserzuflussraten stützt. Unsicherheiten hinsichtlich der Quantifizierung der lokalen Grundwassereintritte ergaben sich aufgrund der räumlichen Variabilität der ${ }^{222} R n$-Endmember-Aktivitäten des Grundwassers. Im Untersuchungsgebiet lagen die gemessenen ${ }^{222} R n$-Aktivitäten des Grundwassers zwischen 1515 und $7540 \mathrm{~Bq} \mathrm{~m} \mathrm{~m}^{-3}$ und waren aufgrund der hohen Anteile an neogenen- und pleistozänen Sanden vergleichsweise gering. Je nach verwendeter ${ }^{222} R n$-Endmember-Aktivität (Median bzw. 25\%- und 75\%-Quantil) resultierten unterschiedlich hohe Grundwasserzuflussraten für die einzelnen Flussabschnitte. Bereiche mit präferenziellem Grundwassereintritt entlang der Spreewitzer Rinne konnten jedoch für alle durchgeführten Szenarien, auch unter Verwendung des $25 \%$ - bzw. $75 \%$-Quantil, bestätigt werden.

Für beide Fließgewässer konnte nachgewiesen werden, dass der präferenzielle Grundwasserzufluss entlang der Spreewitzer Rinne, und der damit verbundene diffuse Eintrag von $\mathrm{Fe}^{2+}$, die Haupt-Eisen-Quelle darstellt. Genau für diese Flussabschnitte entlang der Spreewitzer Rinne wurden in der Vergangenheit bereits hohe gelöste $\mathrm{Fe}^{2+}$-Konzentrationen festgestellt (Uhlmann et al. 2012; Benthaus et al. 2015). Als Quellen der $\mathrm{Fe}^{2+}$-Belastung wurde dabei primär der pleistozäne Grundwasserleiter der Spreewitzer Rinne sowie sekundär die Innenkippe Burghammer und die AuBenhalde Burghammer identifiziert (Uhlmann et al. 2012). Das hohe Eisen-Potenzial der Spreewitzer Rinne in Verbindung mit dem hohen präferenziellen Grundwasserzufluss in diesen Flussabschnitten, sorgt in beiden Fließgewässern für einen abrupten Anstieg der gelösten $\mathrm{Fe}^{2+}$-Konzentrationen. Dies konnte optisch mit der für $\mathrm{Fe}(\mathrm{OH})_{3 s}$ typisch rotbräunlichen Färbung des Gewässers bzw. des Sediments bestätigt werden (siehe Abb. A2-A4 im Anhang).

Eisen liegt aufgrund geringer $\mathrm{pH}-$ Werte und anoxischer Bedingungen im Grundwasser nahezu ausschließlich als zweiwertig gelöstes $\mathrm{Fe}^{2+}$ vor. Der diffuse Eintrag von $\mathrm{Fe}^{2+}$ durch das Grundwasser in die Spree bzw. die Kleine Spree wird aufgrund des hohen Eisen-Potenzials der Spreewitzer Rinne bei rückläufigen Frachten wohl noch weitere 50-100 Jahre anhalten (Uhlmann et al. 2012). Oberirdische Zuflüsse in die Spree bzw. die Kleine Spree aus 
Tagbauresteseen, wie dem Scheibesee und Bernsteinsee (Kleine Spree) sowie dem Schwarzen Schöps (Spree), erhöhen die $\mathrm{Fe}^{2+}$-Fracht in den Fließgewässern aufgrund der geringen $\mathrm{Fe}^{2+}$-Konzentrationen nicht signifikant. Um der durch die $\mathrm{FeS}_{2}$-Verwitterung bedingten Versauerung in den Tagebaurestseen entgegen zu wirken, werden regelmäßig Kalkungsmaßnahmen durchgeführt. Dabei steigt der pHWert und das gelöste $\mathrm{Fe}^{2+}$ präzipitiert in Form von $\mathrm{Fe}(\mathrm{OH})_{3 s}$, während die gelöste $\mathrm{Fe}^{2+}$-Konzentration sinkt (Bengtsson et al. 1980; Klapper et al. 1996). Für die Tagebaurestseen Scheibesee, Bernsteinsee wurde ein Eisenrückhalt $\left[\mathrm{Fe}_{i m m}\right]$ zwischen 2 und 6 Tonnen/Tag und für den Bärwalder See ein Rückhalt zwischen 14-16 Tonnen/Tag basierend auf Gl. 7 bestimmt.

Die Quantifizierung des immobilisierten Eisens in Form von $\mathrm{Fe}(\mathrm{OH})_{3 s}$ zeigte, dass große Mengen an Eisen aus der $\mathrm{FeS}_{2}$-Verwitterung im Einzugsgebiet zurückgehalten werden und nur ein geringer Anteil $(<1 \%)$ in gelöster Form als $\mathrm{Fe}^{2+}$ bzw. als suspendiertes kolloidales $\mathrm{Fe}(\mathrm{OH})_{s}$ das Einzugsgebiet verlässt. Für das gesamte untersuchte Einzugsgebiet der Spree wurde eine zurückgehaltene Menge an Eisen von insgesamt 109-120 Tonnen/Tag ermittelt. Primäre Senken für Eisen im Einzugsgebiet sind 1) die Tagebaurestseen, bei denen durch künstliches Kalken die Präzipitation von $\mathrm{Fe}(\mathrm{OH})_{3 s}$ gefördert wird, 2) die Flussbettsedimente, an deren Grenzschicht zum Aquifer das anoxische Grundwasser mit Sauerstoff in Berührung kommt und $\mathrm{Fe}(\mathrm{OH})_{3 s}$ gebildet wird, und 3) oxische Bereiche im Aquifer, in denen sich $\mathrm{Fe}(\mathrm{OH})_{3 s}$ bilden kann. Die durchgeführte Quantifizierung der im Einzugsgebiet der Spree/Kleinen Spree zurückgehaltenen Menge an Eisen basiert auf der Annahme, dass 1) sich im Gegensatz zu gelöstem $\mathrm{Fe}^{2+}, \mathrm{SO}_{4}{ }^{2-}$ annähernd wie ein konservativer Stoff im Einzugsgebiet verhält und 2) die dominante Quelle, sowohl für $\mathrm{Fe}^{2+}$ als auch $\mathrm{SO}_{4}{ }^{2-}$, die $\mathrm{FeS}_{2}-$ Verwitterung darstellt. Insbesondere für den Altarm konnte gezeigt werden, dass letztere Annahme, aufgrund des stöchiometrischen Verhältnisses zwischen $\mathrm{Fe}^{2+}$ und $\mathrm{SO}_{4}{ }^{2-}$, nahezu perfekt erfüllt wird.

Die Annahme für das konservative Transportverhalten von $\mathrm{SO}_{4}{ }^{2-}$ im Einzugsgebiet, auf dem die Quantifizierung des immobilisierten Eisens basiert, könnte aufgrund von ablaufenden $\mathrm{SO}_{4}{ }^{2-}$-Reduktionsprozessen teilweise nicht zutreffen. Im Grundwasserleiter kann $\mathrm{SO}_{4}^{2-}$-Reduktion in anoxischen Mikronischen stattfinden, die lokal einen hohen Anteil von bioverfügbarem organischen Kohlenstoff aufweisen (Massmann et al. 2003; Scholl et al. 2006). Ähnliche Abbaumechanismen, bei denen $\mathrm{SO}_{4}{ }^{2-}$ unter anoxischen Bedingungen reduziert wird, finden in vorfluternahen Feuchtgebieten statt (Whitmire und Hamilton 2005; Frei et al. 2012). Für das Einzugsgebiet der Spree bzw. der Kleinen Spree kann die $\mathrm{SO}_{4}^{2-}$-Reduktion im Aquifer bzw. in Feuchtgebieten nicht ausgeschlossen werden. Für Bereiche im Einzugsgebiet, wo $\mathrm{SO}_{4}{ }^{2-}$ teilweise reduziert wird, wird der quantifizierte Eisenrückhalt tendenziell unterschätzt, da ein Teil des $\mathrm{SO}_{4}{ }^{2-}$ nicht den Vorfluter erreicht und deshalb nicht in die Bilanzierung eingeht. Aufgrund der sehr hohen $\mathrm{SO}_{4}{ }^{2-}$-Frachten in der Spree/Kleinen Spree gehen wir allerdings davon aus, dass dieser Anteil nicht wesentlich die Bilanzierung beeinflusst.

\section{Zusammenfassung und Fazit}

Basierend auf dem durchgeführten ${ }^{222} R n$-Monitoring konnte nachgewiesen werden, dass sich der lokale Austausch zwischen der Kleinen Spree bzw. der Spree und dem Aquifer räumlich stark unterscheidet. Insbesondere im Bereich der Spreewitzer Rinne kommt es entlang beider Fließgewässer zur Ausprägung von Bereichen mit präferenziellem Grundwasserzufluss, durch die auch große Mengen an Eisen in beide Fließgewässer gelangen. Die Bilanzierung des zurückgehaltenen Eisens ergab enorme Mengen an $\mathrm{Fe}(\mathrm{OH})_{3 s}$, die im Einzugsgebiet der Spree zurückgehalten werden. Als Teil dieser Studie erwies sich das vorgestellte ${ }^{222} R n$ Verfahren als verlässliche und einfach durchzuführende Insitu-Methode zur qualitativen und quantitativen Erfassung lokaler Grundwasserzuflüsse. Das ${ }^{222} R n$-Verfahren eignet sich insbesondere für die Bestimmung der Grundwasserzuflüsse auf der größeren Skala, wie etwa Einzugsgebiete oder ganzer Flussnetze. Ähnliche ${ }^{222} R n$-Studien für die Quantifizierung von lokalen Grundwasserzuflüssen wurden in der Vergangenheit bereits für unterschiedlich große Fließgewässer, wie etwa dem Roten Main $\left(\sim 1 \mathrm{~m}^{3} \mathrm{~s}^{-1}\right)$ (Frei und Gilfedder 2015; Pittroff et al. 2017), der Salzach $\left(\sim 250 \mathrm{~m}^{3} \mathrm{~s}^{-1}\right)$ (Frei und Gilfedder 2015) oder der Vilqué $\left(\sim 101 \mathrm{~s}^{-1}\right)$ (Frei et al. 2019), einem kleinen Fluss/Bach in der Bretagne (Frankreich) durchgeführt. Das Verfahren eignet sich insbesondere gut, wenn in den entsprechenden Einzugsgebieten flussnahe Grundwassermessstellen vorhanden sind, über die sich die für den Ansatz notwendigen ${ }^{222} R n$-Endmemberkonzentrationen ermitteln lassen. Das für die quantitative Auswertung der ${ }^{222} R n$-Messungen notwendige Massenbilanzmodell FINIFLUX ist frei verfügbar unter http://www.hydro.uni-bayreuth.de/hydro/de/software/ software/software_dl.php?id_obj=129191.

Danksagung Dieses Projekt wurde unterstützt von dem Bayerischen Netzwerk für Klimaforschung bayklif (www.bayklif.de). Darüber hinaus möchten wir uns auch bei den anonymen Gutachtern dieser Studie bedanken.

Funding Open Access funding provided by Projekt DEAL.

Open Access Dieser Artikel wird unter der Creative Commons Namensnennung 4.0 International Lizenz veröffentlicht, welche die Nutzung, Vervielfältigung, Bearbeitung, Verbreitung und Wiedergabe in jeglichem Medium und Format erlaubt, sofern Sie den/die ursprünglichen Autor(en) und die Quelle ordnungsgemäß nennen, einen Link 
zur Creative Commons Lizenz beifügen und angeben, ob Änderungen vorgenommen wurden.

Die in diesem Artikel enthaltenen Bilder und sonstiges Drittmaterial unterliegen ebenfalls der genannten Creative Commons Lizenz, sofern sich aus der Abbildungslegende nichts anderes ergibt. Sofern das betreffende Material nicht unter der genannten Creative Commons Lizenz steht und die betreffende Handlung nicht nach gesetzlichen Vorschriften erlaubt ist, ist für die oben aufgeführten Weiterverwendungen des Materials die Einwilligung des jeweiligen Rechteinhabers einzuholen.

Weitere Details zur Lizenz entnehmen Sie bitte der Lizenzinformation auf http://creativecommons.org/licenses/by/4.0/deed.de.

\section{Literatur}

Bengtsson, B., Dickson, W., Nyberg, P.: Liming acid lakes in Sweden. J. Hum. Environ. 34-36 (1980)

Benthaus, F.C., Uhlmann, W., Totsche, O.: Investigation and strategy on iron removal from water courses in mining induced areas. 10thInternational Conference on Acid Rock Drainage \& IMWA Annual Conference. (2015)

Bilek, F., Moritz, F., Albinus, S.: Iron-hydroxide-removal from mining affected rivers. Mining meets water-conflicts and solutions. IMWA Internaional Mine Water Association, Leipzig, S. 151-158 (2016)

Cartwright, I., Gilfedder, B.: Mapping and quantifying groundwater inflows to Deep Creek (Maribyrnong catchment, SE Australia) using 222Rn, implications for protecting groundwater-dependant ecosystems. Appl. Geochem. 52, 118-129 (2015)

Cook, P.G.: Estimating groundwater discharge to rivers from river chemistry surveys. Hydrol. Process. 27(25), 3694-3707 (2013)

Cook, P.L.M., Wenzhöfer, F., Rysgaard, S., Galaktionov, O.S., Meysman, F.J.R., Eyre, B.D., Cornwell, J., Huettel, M., Glud, R.N.: Quantification of denitrification in permeable sediments: Insights from a two-dimensional simulation analysis and experimental data. Limnol. Oceanogr. Methods 4(9), 294-307 (2006)

Doherty, J., Brebber, L., Whyte, P.: PEST: Model-independent parameter estimation. Watermark Computing, Corinda, S. 122 (1994)

Edwards, K.J., Goebel, B.M., Rodgers, T.M., Schrenk, M.O., Gihring, T.M., Cardona, M.M., Mcguire, M.M., Hamers, R.J., Pace, N.R., Banfield, J.F.: Geomicrobiology of pyrite (FeS2) dissolution: case study at Iron Mountain, California. Geomicrobiol. J. 16(2), 155-179 (1999)

Fleischhammel, P., Schoenheinz, D., Grünewald, U.: Bergbaufolgeseen in der Lausitz-naturwissenschaftliche und administrative Herausforderungen bei ihrer Integration in die Gewässerlandschaft und die flussgebietsbezogene Bewirtschaftung. STR10/10., S. $29(2010)$

Frei, S., Gilfedder, B.S.: FINIFLUX: An implicit finite element model for quantification of groundwater fluxes and hyporheic exchange in streams and rivers using radon. Water Resour. Res. 51(8), 6776-6786 (2015)

Frei, S., Durejka, S., Le Lay, H., Thomas, Z., Gilfedder, B.S.: Quantification of hyporheic nitrate removal at the reach scale: exposure times versus residence times. Water Resour. Res. 55(11), 9808-9825 (2019)

Frei, S., Fleckenstein, J.H., Kollet, S.J., Maxwell, R.M.: Patterns and dynamics of river-aquifer exchange with variably-saturated flow using a fully-coupled model. J. Hydrol. Reg. Stud. 375(3-4), 383-393 (2009)

Frei, S., Knorr, K.H., Peiffer, S., Fleckenstein, J.H.: Surface microtopography causes hot spots of biogeochemical activity in wetland systems: a virtual modeling experiment. J. Geophys. Res. 117(G4) (2012). https://doi.org/10.1029/2012JG002012

Gilfedder, B.S., Cartwright, I., Hofmann, H., Frei, S.: Explicit modeling of radon-222 in hydroGeosphere during steady state and dynamic transient storage. Ground Water 57(1), 36-47 (2019). https://doi.org/10.1111/gwat.12847

Gilfedder, B.S., Frei, S., Hofmann, H., Cartwright, I.: Groundwater discharge to wetlands driven by storm and flood events: quantification using continuous radon-222 and electrical conductivity measurements and dynamic mass-balance modelling. Geochim. Cosmochim. Acta 165, 161-177 (2015)

Gleisner, M., Herbert Jr, R.B.: Sulfide mineral oxidation in freshly processed tailings: batch experiments. J. Geochem. Explor. 76(3), 139-153 (2002)

Hüttl, R.F.: Ecology of post strip-mining landscapes in Lusatia, Germany. Environ. Sci. Policy 1(2), 129-135 (1998)

Klapper, H., Geller, W., Schultze, M.: Abatement of acidification in mining lakes in Germany. Lakes Reserv. Res. Manag. 2(1-2), 7-16 (1996)

Kruspe, R., Neumann, J., Opitz, M., Theiss, S., Uhlmann, W., Zimmermann, K.: Fließgewässerorganismen und Eisen: Qualitative und quantitative Beeinflussungen von Fließgewässerorganismen durch Eisen am Beispiel der Lausitzer Braunkohlenfolgelandschaft. Schriftenreihe des LfULG Sachsen 35/2014. (2014)

Massmann, G., Tichomirowa, M., Merz, C., Pekdeger, A.: Sulfide oxidation and sulfate reduction in a shallow groundwater system (Oderbruch Aquifer, Germany). J. Hydrol. 278(1-4), 231-243 (2003)

Meyer, G., Waschkies, C., Hüttl, R.F.: Investigations on pyrite oxidation in mine spoils of the Lusatian lignite mining district. Plant Soil 213(1-2), 137-147 (1999)

Moses, C.O., Nordstrom, D.K., Herman, J.S., Mills, A.L.: Aqueous pyrite oxidation by dissolved oxygen and by ferric iron. Geochim. Cosmochim. Acta 51(6), 1561-1571 (1987)

Nield, S.P., Townley, L.R., Barr, A.D.: A framework for quantitative analysis of surface water-groundwater interaction: flow geometry in a vertical section. Water Resour. Res. 30(8), 2461-2475 (1994)

Pham, A.N., Rose, A.L., Feitz, A.J., Waite, T.D.: Kinetics of Fe (III) precipitation in aqueous solutions at $\mathrm{pH} 6.0-9.5$ and $25 \mathrm{C}$. Geochim. Cosmochim. Acta 70(3), 640-650 (2006)

Pittroff, M., Frei, S., Gilfedder, B.S.: Quantifying nitrate and oxygen reduction rates in the hyporheic zone using $222 \mathrm{Rn}$ to upscale biogeochemical turnover in rivers. Water Resour. Res. 53(1), 563-579 (2017)

Scholl, M.A., Cozzarelli, I.M., Christenson, S.C.: Recharge processes drive sulfate reduction in an alluvial aquifer contaminated with landfill leachate. J. Contam. Hydrol. 86(3-4), 239-261 (2006)

Schultze, M., Pokrandt, K.-H., Hille, W.: Pit lakes of the central German lignite mining district: creation, morphometry and water quality aspects. Limnol. Manag. Inland Waters 40(2), 148-155 (2010)

Schwarzmüller, H., Menz, C.: WELLMA-2 Synthesis report (2013)

Uhlmann, W., Theiss, S., Nestler, W., Zimmermann, K., Claus, T.: Weiterführende Untersuchungen zu den hydrochemischen und ökologischen Auswirkungen der Exfiltration von eisenhaltigem, saurem Grundwasser in die Kleine Spree und in die Spree. IWB, Dresden (2012)

Ulrich, C., Bannehr, L., Hupfer, M., Schulze, M.: Entwicklung von Indizes zur Ableitung der Eisenkonzentration verockerter Fließgewässer mittels hyperspektraler VNIR-und SWIR-Daten. Publ. DGPF 28, 558-571 (2019)

Whitmire, S.L., Hamilton, S.K.: Rapid removal of nitrate and sulfate in freshwater wetland sediments. J. Environ. Qual. 34(6), 2062-2071 (2005)

Woessner, W.W.: Stream and fluvial plain ground water interactions: rescaling hydrogeologic thought. Groundwater 38(3), 423-429 (2000)

Hinweis des Verlags Der Verlag bleibt in Hinblick auf geografische Zuordnungen und Gebietsbezeichnungen in veröffentlichten Karten und Institutsadressen neutral. 